
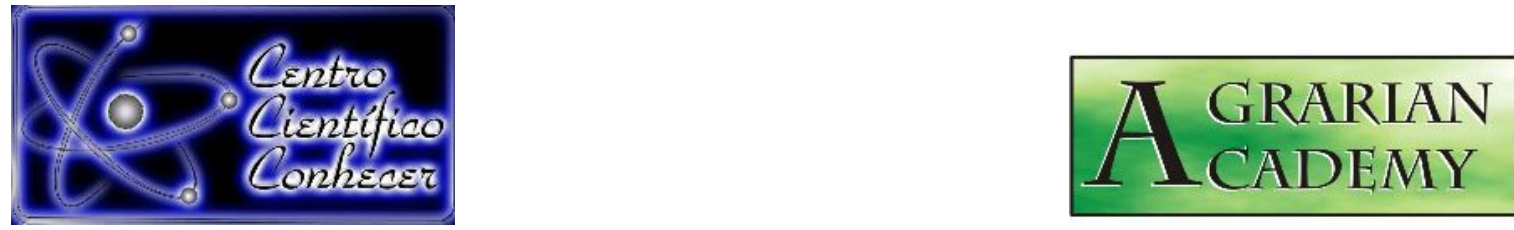

\title{
USO DE EXTRATOS AQUOSOS (NIM, EUCALIPTO E FUMO) NO CONTROLE DO CASCUDINHO (Alphitobius diaperinus)
}

Andressa Graebin Ferreira ${ }^{1}$; Jhonny Kelvin Dias Martins ${ }^{2}$; Weverton Peroni Santos ${ }^{1}$; Daniela Odino Rodrigues ${ }^{1}$; Marina Conceição do Carmo

${ }^{1}$ Graduando (a) do curso de Agronomia, Fundação Universidade Federal de Rondônia - UNIR (andressagraebiin@gmail.com) Rolim de Moura, Brasil.

${ }^{2}$ Engenheiro Agrônomo pela Universidade Federal de Rondônia - UNIR Rolim de Moura, Brasil.

Recebido em: 14/07/2018 - Aprovado em: 28/07/2018 - Publicado em: 31/07/2018 DOI: 10.18677/Agrarian_Academy_2018a43

\begin{abstract}
RESUMO
A avicultura brasileira é uma atividade de grande importância econômica a cada dia vem se expandido. Um dos principais problemas da avicultura é a presença de populações elevadas do cascudinho-dos-aviários, (Alphitobius diaperinus). O uso de extratos vegetais no controle das pragas vem crescendo positivamente, devido a alguns fatores como exigências dos consumidores em ter à disposição produtos mais saudáveis. Diante disso objetivou-se avaliar o uso de extratos no controle do cascudinho. $O$ experimento foi realizado no laboratório de entomologia agrícola na fazenda experimental da Fundação Universidade Federal de Rondônia (UNIR), em Rolim de Moura- RO. Usou-se 0 delineamento experimental inteiramente casualizado em esquema fatorial $3 \times 5$ com cinco repetições, o primeiro fator três extratos aquosos sendo estes de nim (Azadirachta indica), eucalipto (Eucalyptus spp) e fumo (Nicotiana tabacum) e cinco concentrações $(0 ; 2,5 ; 5 ; 10$; $20 \%$ ). As avaliações foram mortalidade acumulada dos insetos observou-se que o extrato de fumo obteve a maior média controle dos insetos na concentração de $10 \%$.
\end{abstract}

PALAVRAS-CHAVE: Granja avícola, inseticida botânico, praga

\section{USE OF AQUEOUS EXTRACTS (NIM, EUCALYPTUS AND SMOKE) IN THE CONTROL OF CASCUDINHO (Alphitobius diaperinus)}

\footnotetext{
ABSTRACT

The Brazilian poultry industry is an activity of great economic importance and has expanded every day. One of the main problems of poultry farming is the presence of high populations of birdwatchers (Alphitobius diaperinus). The use of plant extracts in pest control has been growing positively, due to some factors such as consumer demands for healthier products. Therefore, the objective of this study was to evaluate the use of extracts in the control of the cascudinho. The experiment was carried out in the laboratory of agricultural entomology at the experimental farm of the Federal University of Rondônia Foundation (UNIR), in Rolim de Moura-RO. A completely AGRARIAN ACADEMY, Centro Científico Conhecer - Goiânia, v.5, n.9; p.437 2018
} 
randomized experimental design was used in a $3 \times 5$ factorial scheme with five replicates. The first factor was three aqueous extracts (Azadirachta indica), eucalyptus (Eucalyptus spp) and tobacco (Nicotiana tabacum) and five concentrations $(0 ; 2.5,5,10,20 \%)$. The evaluations were accumulated mortality of the insects it was observed that the smoke extract obtained a greater control of the insects in the concentration of $10 \%$.

KEYWORDS: Poultry farm, botanical insecticide, pest.

\section{INTRODUÇÃO}

A avicultura brasileira é uma das atividades econômicas reconhecidas mundialmente, nesta as exigências de mercado como volume de produção, desempenho econômico e segurança sanitária contribuíram para torná-la uma das mais organizadas e eficientes do mercado mundial (AMARAL et al., 2014). A criação das aves é feita em galpões padronizados (aviários), em regime de confinamento, o que tornou a atividade intensa e produtiva nos últimos anos. Porém, um dos fatores que podem afetar esse ramo da produção é a presença de pragas da granja, como o besouro Alphitobius diaperinus (cascudinho), um inseto da família Tenebrionidae, originário do oeste africano (GIOLO et al., 2013).

O cascudinho dos aviários é considerado um importante problema mundial no sistema de produção avícola, por infestar os aviários (ALVES et al., 2012). Um dos problemas deste inseto é que ele é um vetor de doenças, afetando negativamente a saúde de aves e humanos, e prejudicando a criação de aves. Conforme Giolo et al. (2013) esses insetos são responsáveis pela redução da produtividade e qualidade da carcaça e consequentemente da rentabilidade do avicultor.

O controle deste inseto é geralmente baseado no uso de inseticidas químicos sintéticos, que às vezes são ineficazes, e é limitado devido a exigência do mercado com relação à toxicidade de resíduos químicos em produtos alimentícios (WOLF et al., 2015), um dos problemas na utilização dos produtos químicos sintéticos é que podem contaminar os tecidos das aves, comprometendo a segurança dos alimentos, e no meio ambiente, além de apresentar custo elevado dos produtos finais.

Com intuito de melhorar o controle, sem deixar resíduo no meio ambiente uma das alternativas é o uso de plantas com ação inseticida (MARTINS et al., 2017), o controle de pragas utilizando métodos alternativos, incluindo os extratos vegetais, pode ser uma importante estratégia no controle (VIEIRA; PERES, 2017).

Conforme Coitinho et al. (2006) os inseticidas vegetais podem ser utilizados de inúmeras formas entre estes o pó e os extratos aquosos ou óleos, o que deve ser levado em consideração é qual parte da planta está sendo utilizada e a forma de preparo. Inseticidas naturais são degradados rapidamente, não persistindo no ambiente; a resistência dos insetos às substâncias apresenta processo lento; são de fácil acesso e não deixam resíduos em alimentos e apresentam baixo custo de produção, tornando-se uma estratégia acessível a pequenos produtores (SANTOS et al., 2013).

Nesse contexto segundo Wolf et al. (2015), a combinação de métodos de controle proporciona, entre outros benefícios, redução do uso de produtos químicos, menor possibilidade de desenvolvimento de populações resistentes, melhoria da saúde humana e animal. No entanto, estudos sobre a utilização de plantas inseticidas para o controle de $A$. diaperinus ainda são escassos. Diante disso, o objetivo deste trabalho foi avaliar o potencial de três extratos aquosos, sendo: nim, eucalipto e fumo, sobre o Alphitobius diaperinus adulto, em condições de laboratório. 


\section{MATERIAL E MÉTODOS}

O experimento foi realizado no laboratório de Entomologia Agrícola na Fazenda Experimental da Fundação Universidade Federal de Rondônia (UNIR), em Rolim de Moura- RO. A fazenda experimental está localizada a $15 \mathrm{~km}$ da cidade, nas

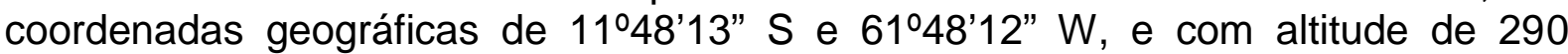
metros acima do nível do mar (LAHORTA, 2014).

Foi usado 0 delineamento experimental inteiramente casualizado em esquema fatorial $3 \times 5$ com cinco repetições, constituído por três extratos aquosos sendo: nim (Azadirachta indica), eucalipto (Eucalyptus spp) e fumo (Nicotiana tabacum) e cinco concentrações $(0 ; 2,5 ; 5 ; 10 ; 20 \%)$.

No experimento foram usados insetos adultos do cascudinho (Alphitobius diaperinus), estes foram coletados em uma granja avícola nas proximidades do município de Rolim de Moura- RO, coletando uma quantidade da "cama" contendo os insetos adultos, sendo conduzidos ao laboratório em bacia plástica coberta com tela.

Utilizou-se no trabalho 15 insetos adultos, foram transferidos para placas de Petri de $9,0 \times 1,5 \mathrm{~cm}$ de diâmetro, revestidas no fundo com papel toalha de folha dupla umedecido com um $\mathrm{mL}$ de água deionizada. Durante a condução do experimento mantidas em condição climatizada a uma temperatura de $24 \pm 2^{\circ} \mathrm{C} \mathrm{e}$ umidade relativa do ar em $75 \pm 3 \%$, como alimento foi oferecido $5 \mathrm{~g}$ de ração para aves.

Os materiais vegetais usados para a produção dos extratos foram: folhas de nim, eucalipto e fumo, esses foram mantidos em estufa à temperatura de $40 \stackrel{\circ}{\circ}$ por 48 horas para secagem e posteriormente, moídos em moinho de faca com peneira de $0,8 \mathrm{~mm}$, obtendo-se pó, com granulação uniforme, e acondicionados em recipientes plásticos para posterior preparação dos extratos.

As concentrações dos extratos aquosos foram determinadas de acordo com Torres et al. (2006) que levaram em consideração a razão da massa/volume $(\mathrm{m} / \mathrm{v})$, misturando o pó do material vegetal em gramas em $100 \mathrm{~mL}$ de água destilada cada um, ficando em repouso por 24 horas, findo esse período, foram feitas filtragens com pano de malha fina para obtenção dos extratos, as soluções foram pulverizadas sobre os insetos pulverizando-os com quatro $\mathrm{mL}$ das concentrações por repetição.

As avaliações e confirmação da mortalidade dos insetos foram realizadas a cada quatro dias tendo um período de avaliação de 20 dias, considerados insetos mortos os indivíduos que não apresentaram nenhuma reação ao toque com pinça metálica. Os resultados significativos foram realizados e a comparação de médias, utilizando-se o teste de Tukey ao nível de $5 \%$ de probabilidade. Para variáveis quantitativas, ajustadas equações de regressão. As análises foram realizadas com o auxílio do programa computacional ASSISTAT ${ }^{\circledR}$, versão 7.7 (SILVA; AZEVEDO, 2016).

\section{RESULTADOS E DISCUSSÃO}

Ao analisar os extratos no controle do inseto na fase adulta, estatisticamente observou-se que o fumo obteve a maior média de mortalidade, alcançando um nível de $18 \%$, já o extrato de eucalipto obteve $4 \%$ sendo a menor taxa de mortalidade (Figura 2). Conforme resultados encontrados por Jacomini et al. (2016) o besouro cascudinho é sensível à ação inseticida do extrato de tabaco assim obtendo em seu trabalho uma taxa de mortalidade superior a $30 \%$.

Segundo Koppad e Shivanna (2010) a nicotina é o principal composto encontrado no extrato do fumo este pode afetar a fecundidade, o comportamento e 
causa efeitos tóxicos nos insetos, a nicotina possui o poder de agir no sistema nervoso central do inseto, se ligando aos receptores. Algumas moléculas de neonicotinoides, o grupo químico de inseticidas sintéticos mais usados no controle de insetos, são derivadas da nicotina do fumo (SPARKS; NAUEN, 2015).

O uso de extrato de nim obteve média de $7 \%$ de mortalidade visto que 0 produto inseticida ativo no nim é a azadiractina eficaz no controle de algumas espécies, resultados diferentes foram encontrados por Giolo et al. (2013) quando observaram que o nim não respondeu ao controle do cascudinho.

O extrato de eucalipto evidenciou uma baixa taxa de mortalidade dos insetos, esse resultado está relacionado ao método utilizado pela extração do extrato. Marcomini et al. (2009) utilizando diferentes métodos e tipos de extratos no controle do cascudinho, verificaram que o extrato de eucalipto não obteve efeito sobre o inseto na fase adulta.

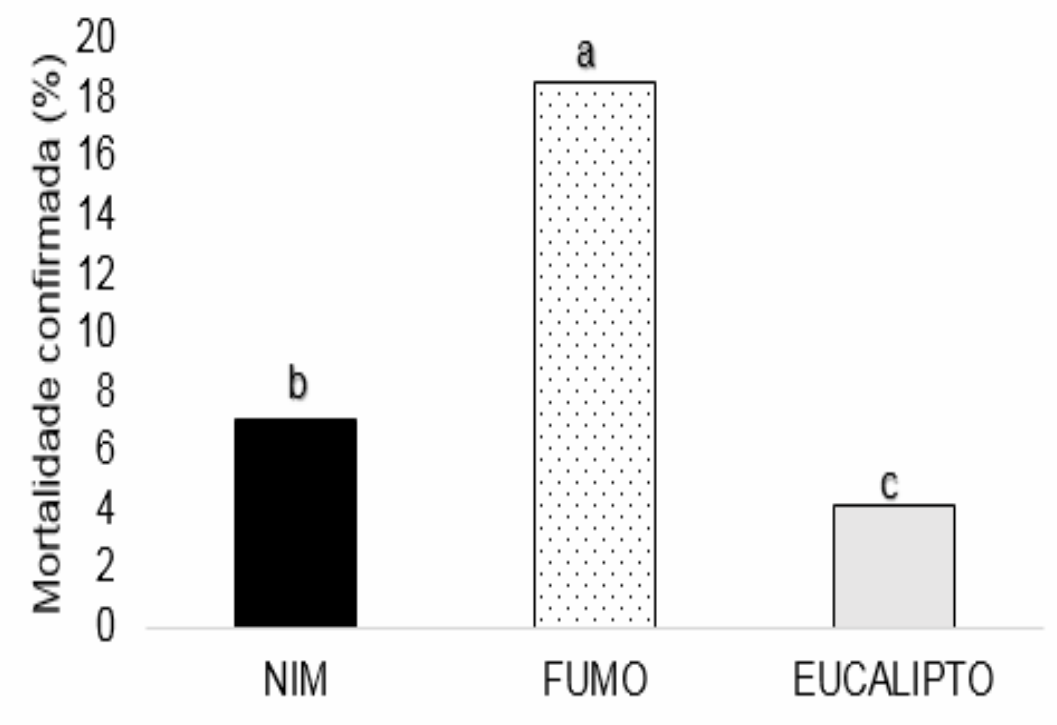

Extratos

FIGURA 1. Mortalidade confirmada dos insetos adultos do cascudinho Alphitobius diaperinus, tratados com extratos aquosos de Nim Fumo e Eucalipto. Médias contendo a mesma letra não diferem entre si pelo teste $\mathrm{F}$ a $5 \%$ de probabilidade.

Com base nas análises, ressalta-se que houve diferença estatística significativa quanto a concentração dos extratos aquosos na porcentagem de mortalidade dos insetos adultos do cascudinho (Figura 2). Evidenciou-se resposta polinomial quadrática, a concentração dos extratos ao nível de $10 \%$ tanto o fumo, nim e o eucalipto foi a que mostrou ter maior eficiência no controle do inseto adulto.

Resultados semelhantes foram encontrados por Marques et al. (2013) testaram diferentes concentrações de extratos de nim obtendo efeito significativo na taxa de mortalidade acima da concentração de 7\%. No entanto o extrato de fumo, independente da concentração mostrou ser mais eficiente na mortalidade dos insetos, comparativamente com os demais extratos e concentrações avaliadas. 
Essa resposta está associada ao principal composto químico que o extrato de fumo contém, que é a nicotina, um alcalóide derivado de várias plantas, principalmente da Nicotiana tabacum. Trata-se de uma toxina que age sobre o sistema nervoso dos insetos, de ação muito rápida e possuindo efeito de contato e de fumigação (MENEZES, 2005). Segundo esse mesmo autor, é uma substância considerada um dos inseticidas botânicos mais tóxicos sendo usada para controlar pulgões, percevejos, vaquinhas, cochonilhas e grilos em plantas frutíferas e hortícolas.

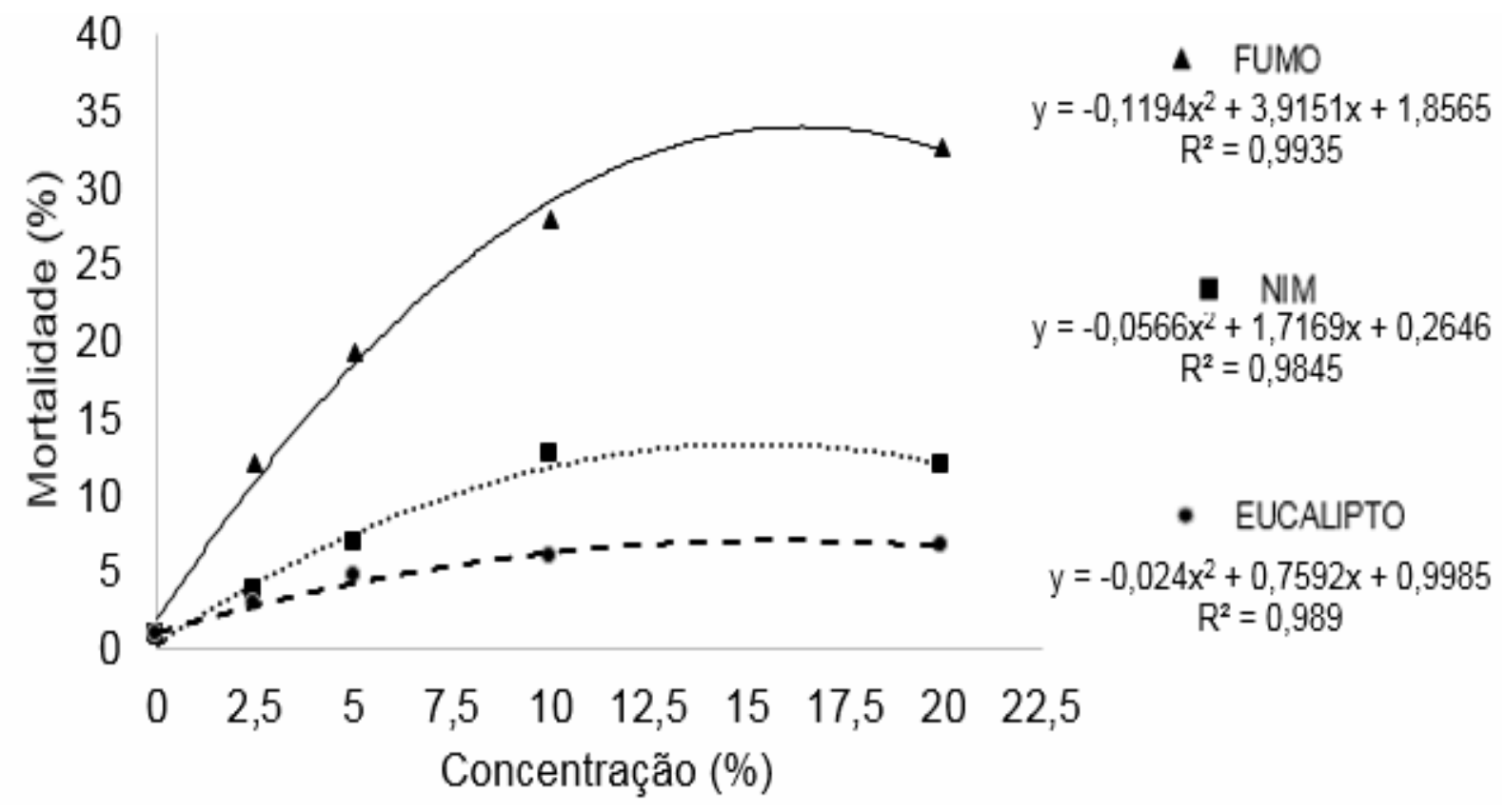

FIGURA 2. Mortalidade de adultos do cascudinho, Alphitobius diaperinus, tratados com extratos aquosos de Fumo, Nim e Eucalipto com diferentes níveis de concentração.

\section{CONCLUSÃO}

O uso dos extratos mostrou-se uma baixa eficiência no controle do cascudinho na fase adulta. Sendo assim, se faz necessário mais estudos na busca por produtos alternativos não químicos.

\section{REFERÊNCIAS}

ALVES, V. M.; ALVES, L. F. A.; LIMA, D. H. U. Atividade da torta de nim sobre adultos do cascudinho dos aviários em condições de laboratório. Ciência Rural, v. 42, n. 5, p. 888-893, 2012. <http://dx.doi.org/10.1590/S0103-84782012005000023> doi: 10.1590/S0103-84782012005000023

AMARAL, P. F. G. P; MARTINS, L. A. E OTUTUMI L. K. Biosseguridade na criação de frangos de corte. Enciclopédia Biosfera, v.10, n.18; p. 664-685, 2014. 
COITINHO, R.L.B.C.; OLIVEIRA, J.V. GONDIM JÚNIOR, M.G.C.; CÂMARA. C.A.G. Atividade inseticida de óleos vegetais sobre Sitophilus zeamais Mots. (Coleoptera: Curculionidae) em milho armazenado. Revista Caatinga, v.19, n.2, p.176-182, 2006.

GIOLO, R.; SENÔ, K. C. A.; FREITAS, A. L.; COSTA, B. O. G.; PELOSI NETTO, F. $J$. Extrato aquoso de Azadirachta indica A. Juss no controle de Alphitobius diaperinus Panzer. Nucleus, v.10, p.165-172, 2013 $<$ http://dx.doi.org/10.3738/1982.2278.920> doi: 10.3738/1982.2278.920.

JACOMINI, D.; TEMPORINI, L. G.; ALVES, L. F. A.; SILVA, E. A. A.; MARINHO, T. C. J. Extrato de tabaco no controle do besouro cascudinho de aviário. Pesquisa Agropecuária brasileira, v.51 n.5, 2016 <http://dx.doi.org/10.1590/S0100204X2016000500032> doi:10.1590/S0100-204X2016000500032

LAHORTA - Laboratório de Horticultura Tropical e Apicultura. KOPPEN-GEIGER, uptader world map of Köppen-Geiger climate classification. Hydrology and Earth System Science., 11, 1633-1644, 2007. Dísponível em: <www.lahorta.acagea.net>. Acesso em: 20. Jun. 2018.

KOPPAD, G.R.; N. SHIVANNA. Effect of nicotine on larval behaviour and fitness in Drosophila melanogaster. Journal of Biopesticides, v. 3, p. 222-226, 2010.

MARCOMINI, A. M.; ALVES, L. F. A.; BONINI A. K.; MERTZ N. R.; DOS SANTOS J. C. atividade inseticida de extratos vegetais e do óleo de nim sobre adultos de alphitobius diaperinus panzer (coleoptera, tenebrionidae). Arquivo Instituto Biológico, v.76, n.3, p.409-416, 2009.

MARQUES, C. R. G.; MIKAMI, A. Y.; PISSINATI, A.; PIVA, LL. B.; SANTOS, O. J. A. P.; VENTURA, M. U. Mortalidade de Alphitobius diaperinus (Panzer) (Coleoptera:Tenebrionidae) por óleos de nim e citronela. Semina: Ciências Agrárias, v. 34 , n. 6 , p. 2565-2574, 2013. <http://dx.doi.org/10.5433/16790359.2013v34n6p2565> doi: 10.5433/1679-0359.2013v34n6p2565

MARTINS, J. K. D.; DA LUZ, S. R. O. T.; DOMINGUES, C .G.; DA SILVA, C. A.; ENCK, B. F. Doses de extratos de alho e pimenta do reino em pó no controle de Sitophilus zeamais em grãos de milho sob condições de armazenamento. Revista Cerrado Agrociências, p. 95-103, dez. 2017.

MENEZES, E. L. A. Inseticidas botânicos: seus princípios ativos, modo de ação e uso agrícola. Seropédica, Rio de Janeiro: Embrapa Agrobiologia, 58p. 2005.

SANTOS, P. L.; PRANDO, M. B.; MORANDO, R.; PEREIRA, G. V. N.; KRONKA, A. Z. Utilização de extratos vegetais em proteção de plantas. Enciclopédia Biosfera, v.9, n.17, p. 2562-2576, 2013.

SILVA, F. de A. S.; AZEVEDO, C. A. V. de. The Assistat Software Version 7.7 and its use in the analysis of experimental data. African Journal of Agricultural Research, v.11, n.39, p.37333740, 2016. <http://dx.doi.org/10.5897/AJAR2016.11522> doi: 10.5897/AJAR2016.11522 
SPARKS, T. C; NAUEN, R. IRAC: Mode of action classification and insecticide resistance management. Pesticide Biochemistry and Physiology, v.121, p. 122128, 2015. <http://dx.doi.org/10.1016/j.pestbp.2014.11.014> doi: 10.1016/j.pestbp.2014.11.014

TORRES, L. A.; BOIÇA JUNIOR, A. L.; MEDEIROS, C. A. M.; BARROS, R. Efeito de extratos aquosos de Azadirachta indica, Melia azedarach e Aspidosperma pyrifolium no desenvolvimento e oviposição de Plutella xylostella. Bragantia, v. 65, n. $\quad 3,2006<$ http://dx.doi.org/10.1590/S0006-87052006000300011> doi: 10.1590/S0006-87052006000300011.

VIEIRA, M. R.; PERES, L. S. Uso de extrato foliar de nim, Azadirachta indica A. Juss, para o controle do pulgão Brevicoryne brassicae (L.) em cultivos de brócolis. Cultura Agronômica, v.26, n.4, p.492-501, 2017.

WOLF, J.; POTRICH, M.; LOZANO, E. R.; GOUVEAA, A.; PEGORINI, C. S. Métodos físicos e químicos combinados para controlar pequenos besouros de larvas de farinha em condições de laboratório, Poultry Science, v, 94, n. 6, p. 11451149, 2015 <https://doi.org/10.3382/ps/pev093> doi: 10.3382/ps/pev093 